\title{
Angiomatous Antrochoanal Polyp Causing Epistaxis and Bone Destruction
}

\author{
${ }^{1}$ Lokesh K Bhama, ${ }^{2}$ Nirmal K Soni
}

\begin{abstract}
Introduction: Angiomatous antrochoanal nasal polyp (AAP) is a rare and distinct variant of a sinonasal polyp, which mimics as a sinonasal malignancy or juvenile nasopharyngeal angiofibroma or a simple antrochoanal polyp owing its property of eroding the bone and causing epistaxis. It is constituting $4-5 \%$ of all nasal polyps
\end{abstract}

Typically, there is extensive vascular proliferation and ectasia with deposition of pseudoamyloid and presence of atypical stromal cells. The extensive reparative and reactive changes are the results of this pseudoneoplastic entity of these polyps. The lesion can often present late, therefore causing both diagnostic and management difficulties. AAP has tendency bleeding and eroding bone. Such rare cases are presented here with a review of the literature about its features and management.

Case report: We describe two cases of angiomatous antrochoanal polyp (AAP) which is a rare variant of sinochoanal polyp. Cases presented with complain of recurrent unilateral epistaxis and nasal obstruction. CT findings revealed erosion of bony walls. The mass in the nasal cavity was reddish pink in color. Endoscopic sinus surgery was performed and a final diagnosis of the angiomatous antrochoanal polyp was made based on the histopathology report.

Management: Complete resection was performed through an endoscopic medial maxillectomy approach while open lateral rhinotomy approach was kept a reserve in case the tumor is not dealt completely via an endoscopic approach. A preoperartive biopsy was taken which was reported as an inflammatory polyp but the surgical specimen was reported as an AAP.

Discussion and conclusion: Very few studies are available on this topic and hence we find little material in the textbook. Being a rare pathological entity, ANP can cause a significant diagnostic dilemma. Clinical, radiological and pathological data are usually complimentary and together provide a definitive diagnosis. The awareness of the existence of such tumors should always be there. Differential diagnosis includes antrochoanal polyp, juvenile angiofibroma, malignancy, papilloma, and hemangioma.

\footnotetext{
${ }^{1}$ ENT Specialist, ${ }^{2}$ Professor

${ }^{1}$ Department of ENT, Dr Bhama ENT Clinic, Mumbai, Maharashtra, India

${ }^{2}$ Department of ENT, University Medical College, Jaipur, Rajasthan, India
}

Corresponding Author: Lokesh K Bhama, ENT Specialist, Department of ENT, Dr Bhama ENT Clinic, Mumbai, Maharashtra, India, e-mail: Ibhama@gmail.com
Keywords: Angiomatous, Antrochoanal, Epistaxis, Polyp, Sinonasal.

How to cite this article: Bhama LK, Soni NK. Angiomatous Antrochoanal Polyp Causing Epistaxis and Bone Destruction. Clin Rhinol An Int J 2018;11(1):25-28.

\section{Source of support: Nil}

\section{Conflict of interest: None}

\section{BACKGROUND}

Angiomatous nasal polyps, are rare and account for approximately $5 \%$ of all inflammatory sinonasal polyps. ${ }^{1}$ Indeed only a very small number have been reported within the literature to date. These polyps present in a variety of ways, often simulating other pathologies including malignancy.

The maxillary sinus is a potential space with a craniofacial skeleton that may involve is a variety of disorders. Many of these disorders share common presenting features also. The antrochoanal polyp is one, that is well known and well-documented disease of the antrum, but its angiomatous variant is not only rare but has unique and unpredictable features, mimicking many other lesions.

We present two cases AAP presenting with a unilateral nasal mass and epistaxis in whom the radiological findings were initially suggestive of malignancy or inverted papilloma, but the histopathological findings subsequently revealed an angiomatous nasal polyp.

\section{CASE REPORT}

\section{Case 1}

A 45-year-old man presented to the ENT department of the hospital with a 2-year history of right-sided recurrent epistaxis and nasal obstruction. He also had complained of facial swelling on the right side since last 5 months. His comorbid medical conditions included hypertension which was in control with antihypertensives. Nasal endoscopy was done which revealed a polypoidal mass filling the nasal cavity and extending into the middle meatus. A biopsy was taken which was reported as an inflammatory polyp. A contrast-enhanced computed tomography (CT) scan of the sinuses was done to assess the extent of the pathology. The CT scan revealed a heterogeneous attenuated large soft 
tissue density lesion in the right maxillary sinus, with the erosion of underlying bone, involving the wall of maxillary sinus, right zygomatic arch, sphenoid wing, adjacent ethmoidal wall, nasal septum, and inferior orbital plate. The lesion was extending anteriorly in subcutaneous maxilla facial region, medially into the right nasal cavity crossing midline into the left nasal cavity and posteriorly extending into temporalis fossa. There was no intracranial or intraorbital extension (Fig 1). An impression of malignant or fungal pathology was made and surgery was planned. An endoscopic surgery was done. Histopathology of a tumor was reported to show fibrocollagenous tissue with dilated vascular spaces and marked congestion with hemorrhage, suggesting the diagnosis of an angiomatous antrochoanal polyp.

Coronal (Fig. 1) and axial (Fig. 2) CT scan cut revealing a completely opacified right maxillary antrum, with further soft tissue thickening within the right nasal cavity enveloping the middle and inferior turbinates, crossing the midline, reaching in the left nasal cavity. There is an erosion of underlying bones, involving the wall of maxil-

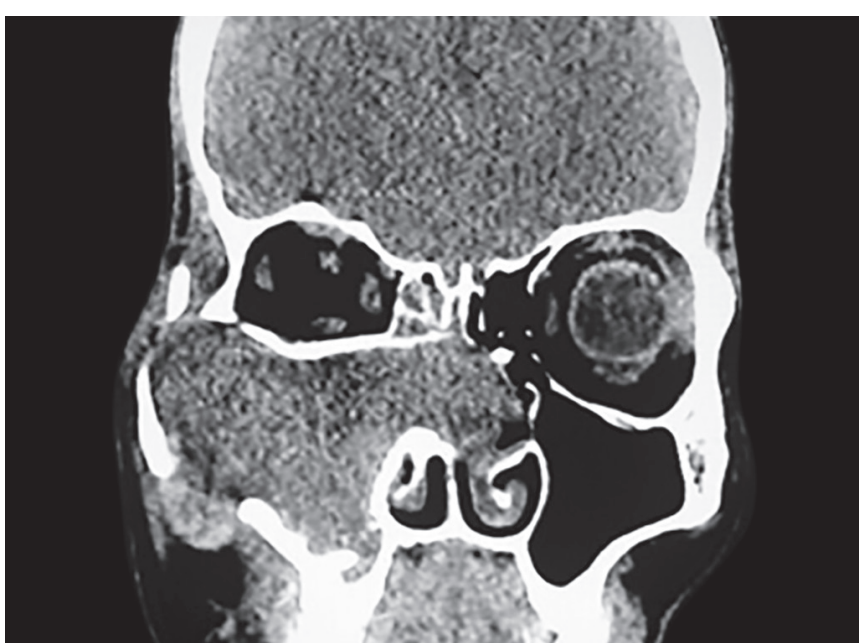

Fig. 1: Bone destruction by angiomatous polyp

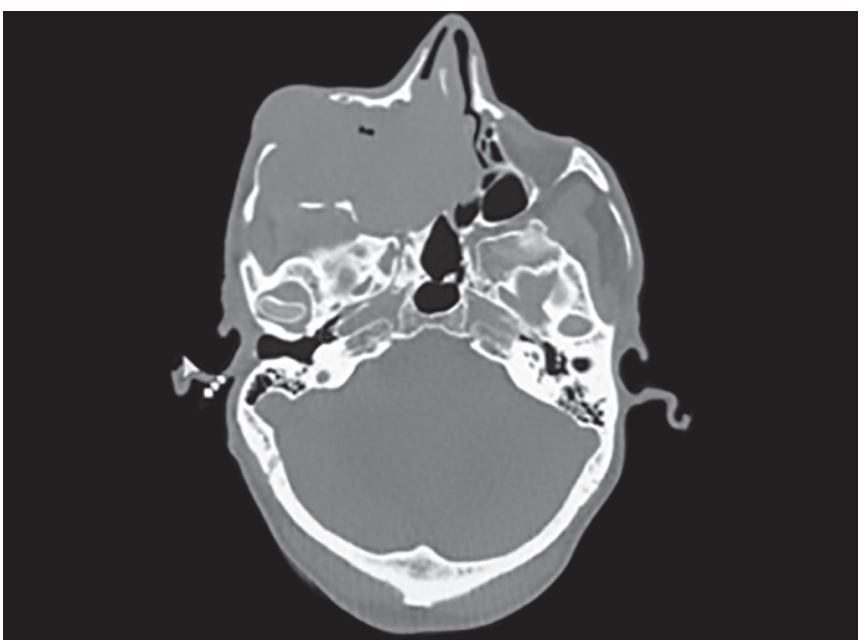

Fig. 2: Axial cuts of CT scan showing destruction of bony walls of sinuses lary sinus, right zygomatic arch, sphenoid wing, adjacent ethmoidal wall, nasal septum, and inferior orbital plate.

The radiological features were consistent with a malignancy. However, the biopsy was reported as simple polyp. Therefore an endoscopic medial maxillectomy was scheduled, and open lateral rhinotomy approach was kept in reserve. It was relatively easy to excise the tissue and the tissue came in pieces. Complete resection of the tumor was achieved by endoscopic approach.

The patient's recovery was uneventful. The histopathology was reported as AAP (Fig. 3).

\section{Case 2}

A 28-year-old female presented to the OPD with complaints of left-sided nasal obstruction of 6 months duration with a history of several episodes of nasal bleeding. There was no other history of any other comorbid illness. A nasal endoscopic examination was done which revealed a reddish polypoidal mass in the left nasal cavity and some blood clots. The mass did not bleed on gentle touch. The septum was grossly central. ACT scan was advised which revealed a homogeneous mass-occupying left maxillary sinus with the destruction of the medial wall of the sinus and involving the anterior and posterior ethmoid cells. The mass was hanging down in the nasopharynx. Endoscopic sinus surgery was performed under general anaethesia, and the mass was removed. Histopathological examination showed numerous thick-walled hyalinized blood vessels embedded in loose fibrous stroma with infiltration by plasma cells, foci of calcification and area of hemorrhage with the covering of pseudostratified columnar ciliated epithelium. On this ground, an impression of the angiomatous antrochoanal polyp was made.

Coronal (Fig. 4) and axial cuts (Fig. 5) CT scan revealing a completely opacified left maxillary with soft tissue thickening within the right nasal cavity enveloping the middle and inferior turbinates and hanging down in the nasopharynx.

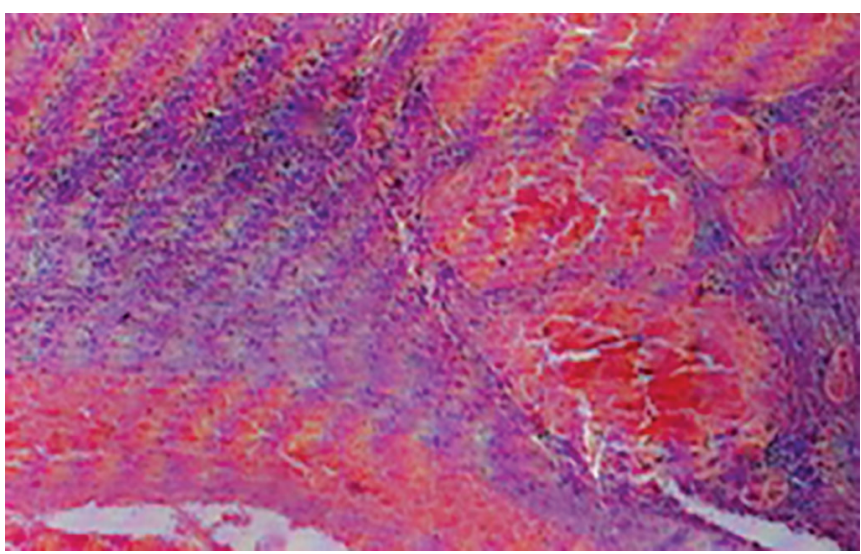

Fig. 3: Photomicrograph showing a well-defined polyp with stromal degenerative hyaline changes stromal hemorrhage 


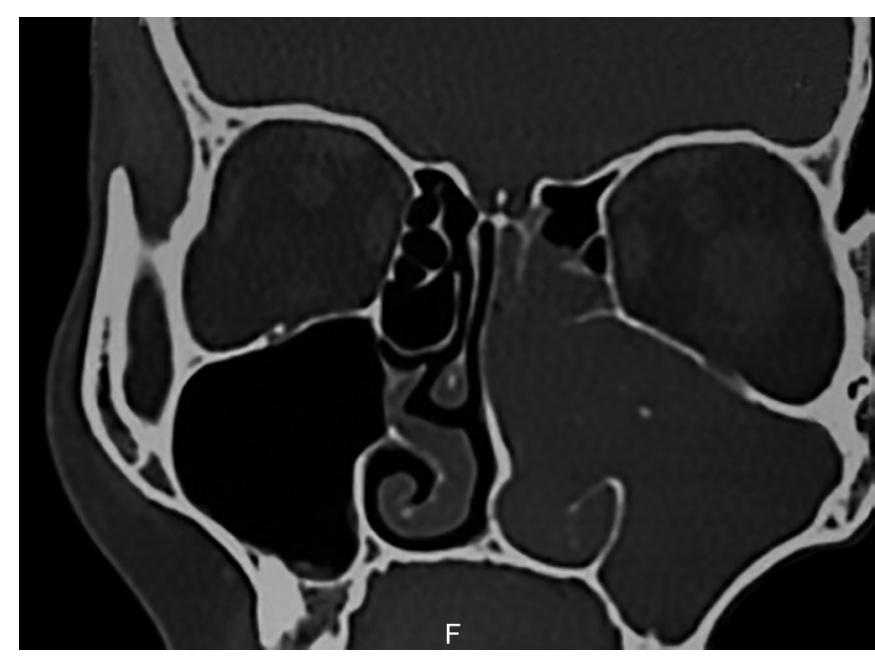

Fig. 4: CT scan showing involvement of multiple paranasal sinuses on left side

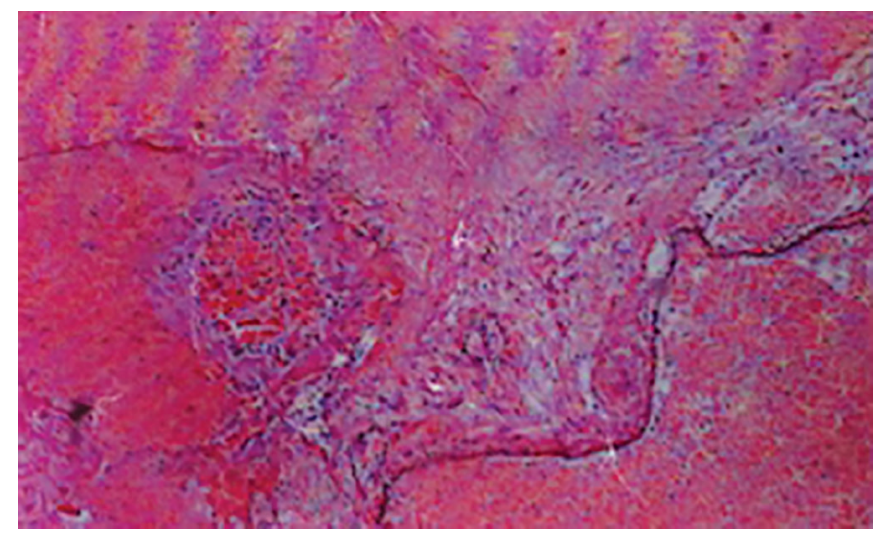

Fig. 6: Histopathology report shows AAP

These radiological features were consistent with a sinonasal inverted papilloma. An endoscopic medial maxillectomy was scheduled and complete excision was done.

The patient's recovery was uneventful. The histopathology (Fig. 6) report was suggestive of an AAP.

\section{DISCUSSION}

Benign inflammatory sinonasal polyps are histologically divided into five main groups: oedematous, glandular, fibrous, cystic and angiectatic (angiomatous). ${ }^{2}$ Angiomatous nasal polyps are rare, accounting for only $4-5 \%$ of all nasal polyps. ${ }^{3}$ Mostly, the present with recurrent epistaxis and have a capacity to widespread bone destruction with deformity, thereby simulating a malignancy. ${ }^{2}$

Angiomatous antrochonal nasal polyps are fibrosed, vascularized nasal polyp, probably a response to a minor trauma. ${ }^{4}$ Batsakis hypothesized that vascular compression of the polyp at the vulnerable site results in infarction followed by reparative changes and neovascularization. ${ }^{4}$ There are four such vulnerable sites: the ostial exit site, the posterior end of the inferior turbinate, the posterior

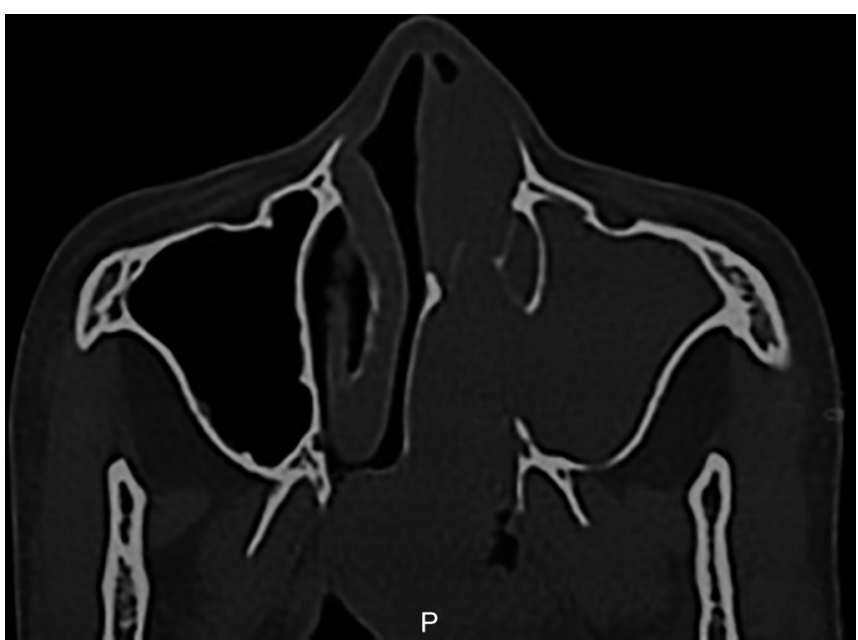

Fig. 5: CT scan showing presence of tumor in sinuses and nasopharynx

choana, and the most dependent part within the nasopharynx..$^{5,6}$

The fragility of the AAP is due to infarction, and hence it is difficult to remove them en bloc. Perivascular pools of eosinophilic material are because of the extravasations of blood components (fibrin, platelets, etc.) through the vessels. Hence a classical histological picture is seen with thin walled vessels embedded in the eosinophilic material (congo red negative), areas of patchy necrosis, scanty inflammatory exudates (mainly the plasma cells) ${ }^{7}$ associated with patchy necrosis and atypical stromal spindle cells (large pleomorphic). ${ }^{8}$

Clinical, histological and radiological differential diagnosis of AAP are antrochoanal polyp, hemangioma, juvenile nasopharyngeal angiofibroma, inverted papilloma and malignancy, In this case, though the endoscopic and gross picture resembled that of a malignancy in first and inverted papilloma in the second case, the existence of recurrent bleeding and destruction of bony wall of the maxillary sinus were against this diagnosis. A suspicion of hemangioma (cavernous/capillary) may arise due to occurrences of recurrent bleeding, but this diagnosis was not supported by radiological findings. Angiomas are usually found at the anterior nasal septum, the turbinate and the vestibule. ${ }^{1}$

Angiomatous antrochoanal nasal polyp (AAP) can easily be confused with juvenile angiofibroma ${ }^{9}$ as the later also presents with unilateral epistaxis and nasal obstruction. This diagnosis is not supported by age in first and age and sex of the second patient. By definition, these polyps originate in the maxillary sinus, extend through the maxillary ostium, and continue posteriorly toward the choanae. The main feature which differentiates AAP from juvenile angiofibroma is that the later show marked enhancement with contrast and may also extend into the pterygopalatine fossa or may have intracranial extenstion. ${ }^{10}$ 
Inverted papillomas are difficult to distinguish from AAP based on clinical, radiological and often histological features. However AAP show more prominent vascular changes, which when present should alert further histological and radiological examination, enabling an accurate diagnosis to be made. ${ }^{2}$

Another differential diagnosis is a malignancy. ${ }^{1}$ Both the lesions show aggressive clinical behavior. Without knowledge of the typical clinical presentation and the complete study of imaging findings, the entity tends to be clinically and radiologically confused with neoplastic processes and even malignancy. ${ }^{11}$ The mass shows minimal enhancement on the edge of the lesions. The edge of ANPs on CT is clear and does not invade the peripheral fat layer. ${ }^{11}$ During surgery, AAP can be excised easily while malignant tumors are not that easy to excise.

\section{CONCLUSION}

Angiomatous antrochoanal polyp is a rare entity which may mimic a variety of lesions of the nose, and paranasal sinuses, careful clinical, radiological, histopathological evaluation, and awareness of its existence can help the surgeon to come out of the diagnostic dilemma. Extensive vascular proliferation, atypical stromal cells, and extracellular amorphous eosinophilic accumulation are typical of ANPs. Because of the high soft-tissue resolution, MR imaging has conspicuous superiority to $\mathrm{CT}$ in reflecting the internal structures of AAP and the involved extent. ${ }^{12}$ Most of the times, these tumors can be excised endoscopically. The surgical goal should be the total excision of ANP and prevention of the recurrence.

\section{REFERENCES}

1. Yfantis HG, Drachenberg CB, Gray W, Papadimitriou JC. Angiectatic nasal polyps that clinically simulate a malignant process: report of 2 cases and review of the literature. Arch Pathol Lab Med 2000;124:406-410.

2. Jayaram S, Svecova N, Biggs TC, et al. Angiomatous Antrochoanal polyp Presenting with epistaxis and bone destruction.

3. Nimbker SA, Sane SY. Histology and histochemistry of nasal polyps. J Postgrad Med 1978;24:231-234.

4. Batsakis J. Tumors of head and neck: Clinical and pathological considerations (2nd ed). Baltimore: Wiliams and Wilkins; 1979, pp. 139-143.

5. Park CS, Noh H, BaeSC, et al. Antrochonal po;yp's varient, the angiomatous nasal polyp: A case report. Korean J Otolaryngo Head and Neck Surg 2006;49(4):443-446.

6. Sheahan P, Crotty PL, Hamilton S, et al. Infarcted angiomatous nasal polyps. Eur Arch Otolaryngol 2005;262:225-230.

7. Sayed RH, Abu-Dief EE. Does antrochoanal polyp present with epistaxis? B-ENT 2007;3(3):145-147.

8. Verma N, Kumar N, Azad R, et al. Angiomatous nasal polyp: A condition difficult to diagnose. Otolaryngology Clinics: An Internatinal J 2011;3(2):93-97.

9. Som P, Cohen B, Sacher M. The angiomatous polyp and the angiofibroma: Two different lesions. Radiology 1982;144: 329-334.

11. Dai LB, Zhou SH, Ruan LX, et al. Correlation of computed tomography with pathological features in angiomatous nasal polyps. PLoS One 2012;7:e53306.

12. Wang $\mathrm{YZ}$, Yang BT, Wang ZC, et al. MR evaluation of sinonasal angiomatous polyp. Am J Neuroradiol 2012;33(4):767-772. 